\title{
Application of interconnected porous hydroxyapatite ceramic block for onlay block bone grafting in implant treatment: A case report (Review)
}

\author{
KOUJI OHTA ${ }^{1}$, MISATO TADA ${ }^{1}$, YOSHIAKI NINOMIYA ${ }^{1}$, HIROKI KATO ${ }^{1}$, FUMI ISHIDA ${ }^{1}$, \\ HITOSHI ABEKURA ${ }^{2}$, KAZUHIRO TSUGA ${ }^{2}$ and MASAAKI TAKECHI ${ }^{1}$
}

${ }^{1}$ Department of Oral and Maxillofacial Surgery, Graduate School of Biomedical and Health Sciences; ${ }^{2}$ Department of
Advanced Prosthodontics, Institute of Biomedical and Health Sciences, Hiroshima University, Hiroshima 734-8553, Japan

Received March 30, 2017; Accepted August 10, 2017

DOI: 10.3892/etm.2017.5224

\begin{abstract}
Autogenous block bone grafting as treatment for alveolar ridge atrophy has various disadvantages, including a limited availability of sufficiently sized and shaped grafts, donor site morbidity and resorption of the grafted bone. As a result, interconnected porous hydroxyapatite ceramic (IP-CHA) materials with high porosity have been developed and used successfully in orthopedic cases. To the best of the author's knowledge, this is the first report of clinical application of an IP-CHA block for onlay grafting for implant treatment in a patient with horizontal alveolar atrophy. The present study performed onlay block grafting using an IP-CHA block to restore bone volume for implant placement in the alveolar ridge area without collecting autogenous bone. Dental X-ray findings revealed that the border of the IP-CHA block became increasingly vague over the 3 -year period, whereas CT scanning revealed that the gap between the block and bone had a smooth transition, indicating that IP-CHA improved the process of integration with host bone. In follow-up examinations over a period of 5 years, the implants and superstructures had no problems. An IP-CHA block may be useful as a substitute for onlay block bone grafting in implant treatment.
\end{abstract}

\section{Introduction}

Alveolar ridge atrophy developing after tooth extraction may result in insufficient bone volume, thus rendering incorrect implant placement from both functional and esthetic viewpoints (1). Autogenous onlay block bone grafts are widely used

Correspondence to: Dr Kouji Ohta, Department of Oral and Maxillofacial Surgery, Graduate School of Biomedical and Health Sciences, Hiroshima University, 1-2-3 Kasumi, Minami-Ku, Hiroshima 734-8553, Japan

E-mail: otkouji@hiroshima-u.ac.jp

Key words: autogenous block bone grafting, implant treatment, interconnected porous hydroxyapatite ceramics for external augmentation in cases of horizontal and vertical alveolar ridge atrophy, as placement on the surface of the host bone can restore bone volume $(2,3)$. Furthermore, several reports have noted that implant treatment with an autogenous onlay block bone graft for horizontal or vertical alveolar ridge atrophy results in bone gain and high rates of implant success (3-6). However, autogenous block bone grafting also has some disadvantages, such as limited availability of grafts with sufficient size and shape, and risk of donor site morbidity, including long-lasting pain, fracture, and nerve damage (7-9). In addition, other problems associated with resorption of the grafted bone during the healing process remain.

Interconnected porous hydroxyapatite ceramic (IP-CHA) materials with high porosity have been developed and used successfully in the field of orthopedics medicine (10). An IP-CHA block, which consists of a porous sintered body composed of hydroxyapatite ceramics with a unique pore structure, is able to undergo extensive incorporation into host bone more rapidly than conventional porous calcium hydroxyapatite ceramic $(10,11)$. We speculated that problems associated with autogenous block bone grafting could be avoided if an IP-CHA block of the same size as an autogenous block bone were to be used for onlay grafting. In our previous study, we used titanium implants in IP-CHA blocks placed on cortical bone surfaces in a rabbit model, which resulted in direct contact between the implant surface and new bone incorporated into the block (12). Those results indicated that use of an IP-CHA block promotes osseointegration of the dental implant from the surface of the host bone. Here, we present the first report of clinical application of an IP-CHA block for onlay grafting in implant treatment in a patient with horizontal alveolar atrophy.

\section{Case report}

The patient was 51-year-old woman, whose left incisor had been extracted at a primary care dental clinic because of caries. Six months later, she was referred to our hospital for consideration of implant treatment for the missing tooth (Fig. 1). Orthopantomograph and computed tomography (CT) images showed horizontal alveolar bone atrophy in the 
anterior region (Fig. 2). Data obtained from the examinations were then transferred to 3D planning software (SimPlant; Materialise Dental NV, Leuven, Belgium) for ideal implant placement (Fig. 3), and those findings showed that the implant thread would be exposed because of the insufficient bone volume in the alveolar ridge. Therefore, we planned to use of an onlay graft with an IP-CHA block to restore bone volume for implant placement in the alveolar ridge area without collecting autogenous block. The virtual planning data were then utilized to create a 3D model by a rapid prototyping machine (Eden 260; Objet Geometries Inc., Rehovot, Israel). An IP-CHA block was fabricated $(6 \times 7 \times 3 \mathrm{~mm}$ in size) by MMT. Co., Ltd., Osaka, Japan, to fit the alveolar ridge of the patient (Fig. 4).

The patient underwent informed consent according to a protocol approved by the Ethical Committee of Hiroshima University Hospital, and onlay grafts and implant placement were performed under general anesthesia in October 2011. A crestal incision and 2 vertical releasing incisions were made, after which the soft tissue flap was raised, and the cortical bone surface was polished using a small round bur to support blood vessel outgrowth. An implant (Replace Tapered groovy NP 3.5x10 mm; Nobel Biocare, Gottenborg, Sweden) was installed into alveolar bone according to the manufacturer's instructions, and the thread remained exposed, as expected (Fig. 5A). Next, 2 small holes for the suture were opened through labial and palate cortical bone on both sides of the thread using a small round bur, with CT imaging employed to avoid injury to the incisive canal, then an absorbable suture was passed through in the labial and palate alveolar ridge via these holes. The IP-CHA block was placed over the exposed thread and fixed to the alveolar ridge with an absorbable suture (Ethicon, Inc., Somerville, NJ, USA) (Fig. 5B). Another incision was made through the periosteum at the base of the flap, thus allowing the tissue to cover the graft without tension, and the flap was sutured. Six months after the procedure, we performed a second operation for abutment connection. Using the same incision as before, subperiosteal dissection of the alveolar bone was performed under local anesthesia. At that time, we observed that the IP-CHA block had become stabilized on the host bone, and no abnormal resorption was found. The ISQ value for the implant obtained at the second operation was found to be increased to $64.6 \pm 0.58$ as compared to that at the first operation $(58.6 \pm 0.58)$. Finally, a healing abutment was exposed above the gingival tissue, and the flap was then sutured. No complications, including infection, abnormal pain and hypoethesia, were observed following surgery.

At 16 months after implant placement with the onlay graft, CT scanning showed that the IP-CHA block had stabilized without abnormal resorption and no problems with the implant were revealed (Fig. 6), thus the final superstructure was placed (Fig. 7). Dental X-ray findings showed that the border of the IP-CHA block had become increasingly vague over the 3 -years period (Fig. 8A-C). CT scan images obtained at 3 years 5 months after onlay grafting showed the IP-CHA block on the alveolar bone, with a smooth transition in the gap between the block and bone, indicating that use of IP-CHA improved the process of integration with host bone (Fig. 8D and E). In the follow-up examinations conducted over 5 years, the implants and superstructures have had no problems.

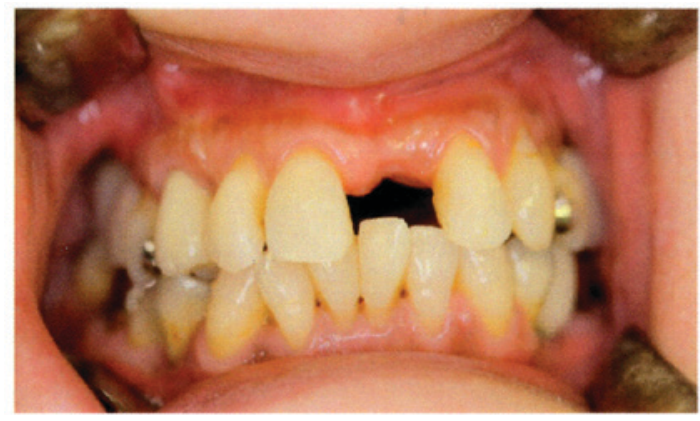

Figure 1. Initial intraoral findings.
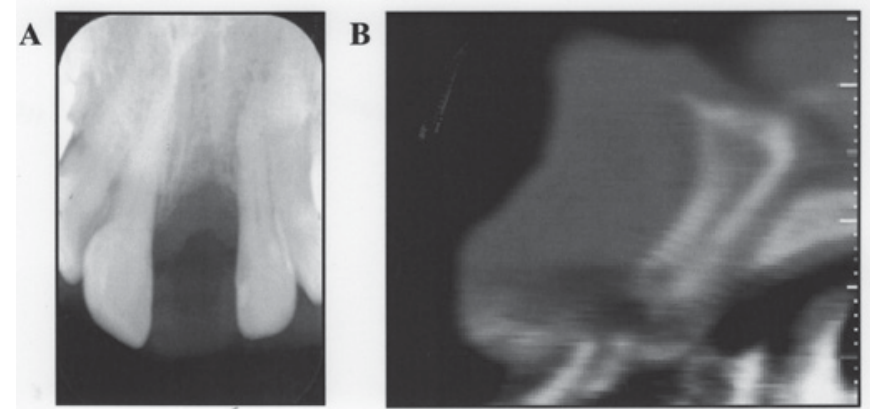

Figure 2. Initial radiographic findings. (A) Dental radiograph (B) Reconstructed sagittal CT scan image.

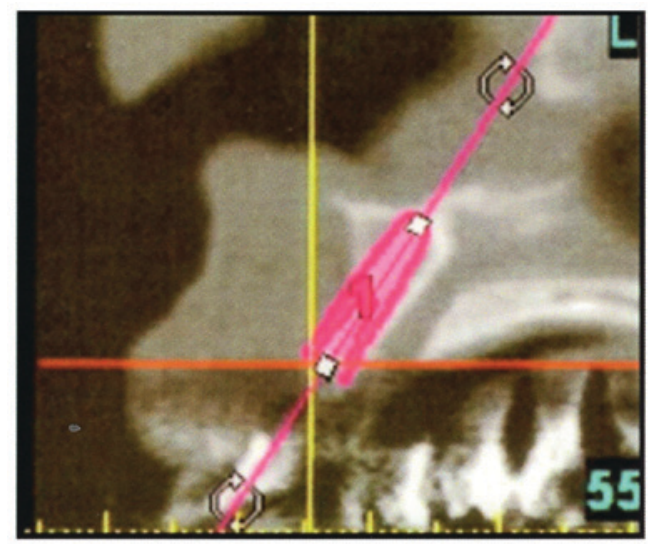

Figure 3. 3D planning analysis for determining ideal implant placement.

\section{Discussion}

Onlay block bone grafts are used for external augmentation of horizontal or vertical alveolar ridge atrophy, with autogenous bone harvested from the mandibular ramus or symphysis used for the graft, as those locations offer the greatest bone volume (13). However, intraoral grafts have been reported to have various drawbacks, such as need for an additional surgical procedure to procure the bone graft material, increased operative time, graft bone limitations, post-operative pain, altered sensation in mandibular teeth, neurosensory disturbances, nerve paresthesia, and mandibular fracture $(8,14)$. In addition, an autogenous bone block usually undergoes extensive resorption during healing (15), 


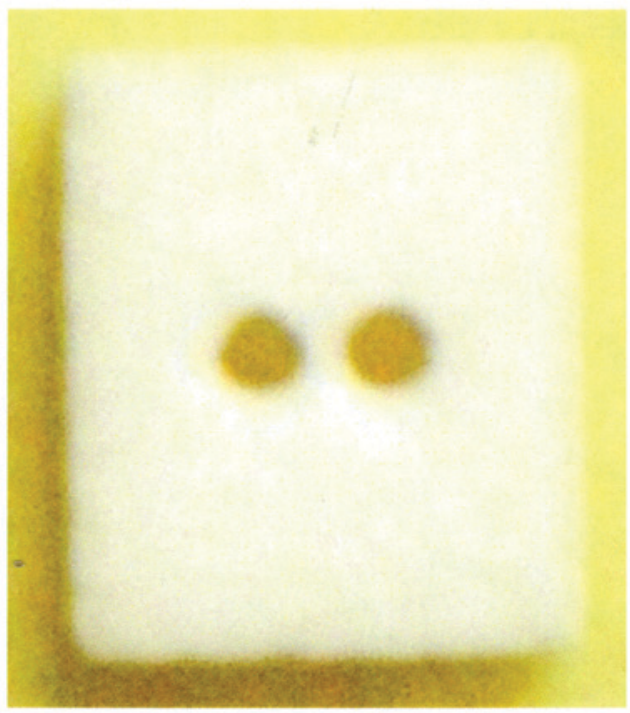

Figure 4. Fabricated IP-CHA block.
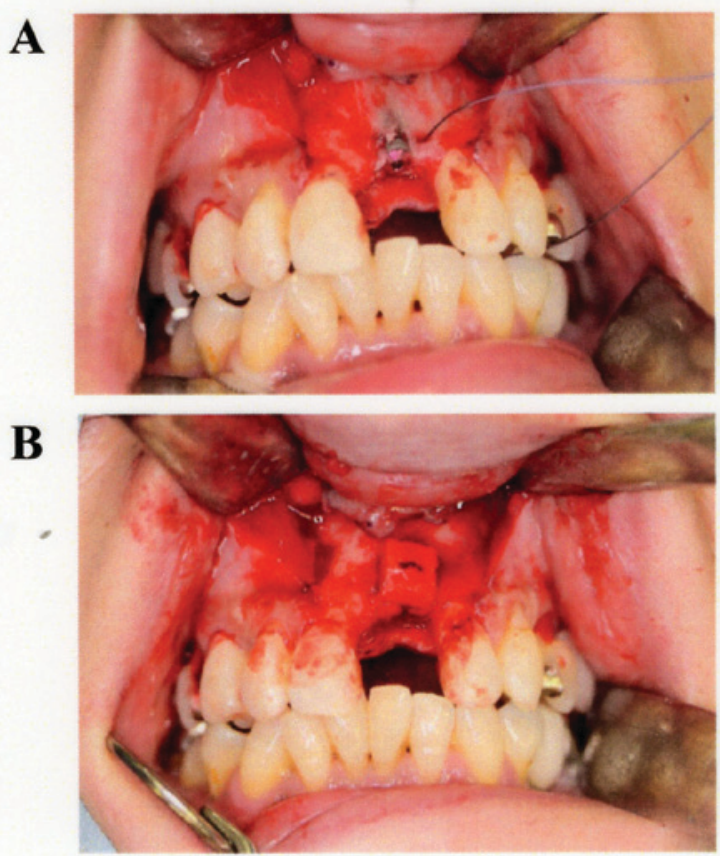

Figure 5. Onlay grafting using an IP-CHA block. (A) The implant was inserted into the alveolar bone, though the thread remained exposed. (B) The IP-CHA block was placed over the exposed thread, and fixed onto the alveolar bone.
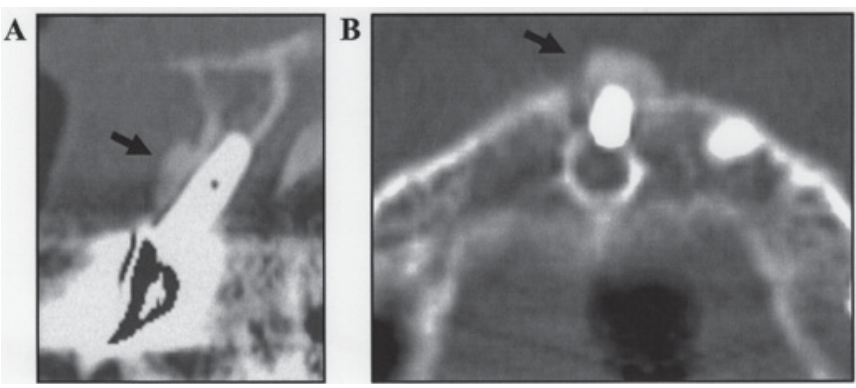

Figure 6. CT scan images at 16 months after onlay block grafting Reconstructed (A) sagittal and (B) axial CT scan images. An IP-CHA block was shown by arrows.

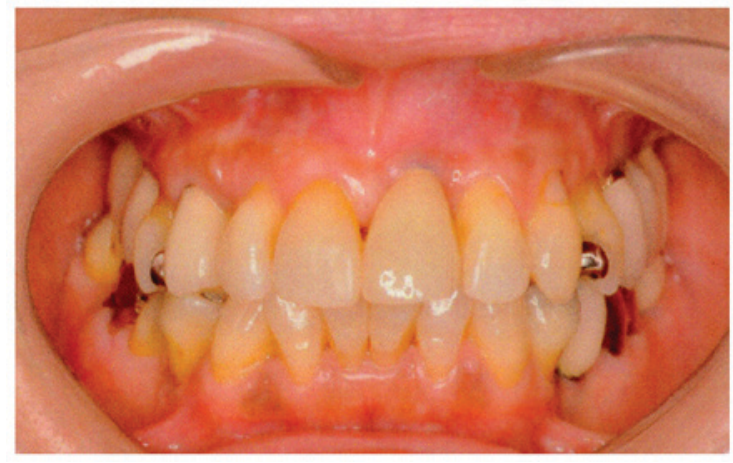

Figure 7. Intraoral view at 16 months after onlay block grafting.
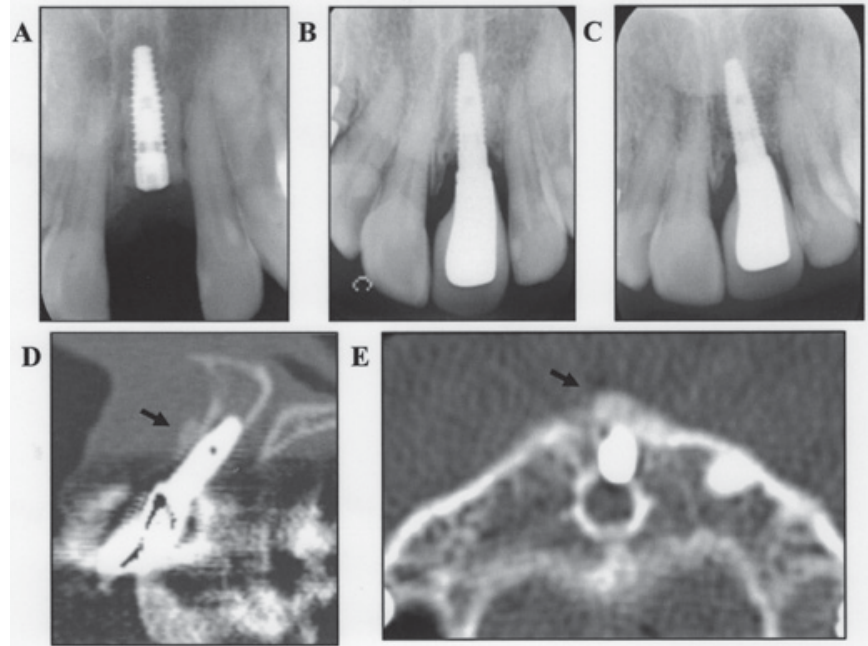

Figure 8. Dental radiograph and CT scan images after onlay block grafting. Dental radiograph obtained (A) immediately, (B) 10 months, and (C) 3 years 5 month after initial surgery. Reconstructed (D) sagittal and (E) axial CT scan images. An IP-CHA block was shown by arrows.

which may result in implant failure from osseointegration loss leading to reduced bone-to-implant contact and an unfavorable outcome.

Hydroxyapatite ceramics (HA) materials have been used as a substitute for bone grafting because the crystalline phase of natural bone is similar to that of HA $(16,17)$, while porous calcium hydroxyapatite ceramics (CHA) materials have been utilized in orthopedic and craniofacial surgery procedures since the 1980s (18). However, few studies have reported cases in which conventional CHA became fully filled by newly formed bone, which may be due to its structure and limited connectivity between pores (19). IP-CHA consists of a porous sintered body made of hydroxyapatite ceramics with a unique pore structure, in which the pores are fully interconnected, likely allowing ingrowth of osteoblasts $(20,21)$. Tamai et al (20) implanted cylindrical blocks made of IP-CHA into femoral condyles of rabbits, and observed mature bone ingrowth in most of the pores within 6 months. IP-CHA has adequate compression strength (10-12 MPa), similar to that of cancellous bone, and was shown to have IP-CHA increased compression strength up to 9 weeks after implantation, reaching approximately $30 \mathrm{MPa}$ (20). It has also been reported that IP-CHA did not show active resorption in clinical applications (10). Since an 
Table I. Reported applications of IP-CHA in oral and maxillofacial surgery cases.

A,

\begin{tabular}{cccccll}
\hline Case & Age $(\mathrm{yrs})$ & Gender & Site & Region & Grafting method & $\begin{array}{l}\text { Autogenous block } \\
\text { bone graft }\end{array}$ \\
\hline 1 & 59 & Female & Maxilla & $\begin{array}{l}\text { Premolar and } \\
\text { molar }\end{array}$ & $\begin{array}{l}\text { Sinus floor } \\
\text { augmentation }\end{array}$ & $\begin{array}{l}\text { Cortical bone block } \\
\text { (10x8 mm) from } \\
\text { maxillary tuberosity } \\
\text { Cortical bone block } \\
\text { (10x15 mm) from } \\
\text { mandibular ramus }\end{array}$ \\
3 & 51 & Male & Maxilla & Incisor & $\begin{array}{l}\text { Onlay block bone } \\
\text { grafting }\end{array}$ & $\begin{array}{l}\text { Onlay block bone } \\
\text { grafting }\end{array}$ \\
\hline
\end{tabular}

$\mathrm{B}$,

Type of IP-CHA

Implant treatment (diameter $\mathrm{x}$ length $\mathrm{mm}$ )

(Refs.)

Granular type $(1-2 \mathrm{~mm})$

Granular type $(1-2 \mathrm{~mm})$

3 implants placed $(4.3 \times 10,4.3 \times 13,5.0 \times 13)$

None

1 implant placed $(4.3 \times 10)$

Block type $(6 \times 7 \times 3 \mathrm{~mm})$

Present case

IP-CHA, interconnected porous hydroxyapatite ceramic.

IP-CHA block can be prefabricated into a specific size and shape to match the alveolar ridge of the patient, application as a substitute autogenous block bone graft for onlay grafting is possible. In the present study, we used an IP-CHA block to overcome disadvantages normally associated with an autogenous bone graft and obtained good results.

Recently, Doi et al (22) reported successful use of IP-CHA as a grafting material for implant treatment in vivo. They examined the effects on bone regeneration of an implant/IP-CHA complex placed directly into femur sockets of dogs as well as implant stability, and found no significant differences in regard to bone implant contact and ISQ values between the complex and control groups at 3-6 months after surgery. In our previous in vivo study, to examine whether an IP-CHA block could be applied as an onlay graft substitute, titanium implants were inserted into IP-CHA blocks placed on the cortical bone surface of the mandibular in rabbits. We observed high levels of new bone formation from the host bone in the pores of the IP-CHA as well as significantly increased ISQ values at 12 weeks after surgery (12). In the present case, the IP-CHA block became stabilized on the host bone, and no abnormal resorption was observed during a second operation performed 6 months after the initial operation. In addition, the ISQ value was increased as compared with that at the first operation. Together, these results show that an IP-CHA block can promote osseoconduction from the surface of the host bone and periosteum, leading to osseointegration of the implant in host bone tissue.

Clinically, IP-CHA is widely used in the field of orthopedic surgery (10). Yoshikawa et al (10) and Shi et al (23) applied IP-CHA granules or blocks as bone substitute for treatment of 59 patients with benign bone tumors and 12 with cystic lesions associated with rheumatoid arthritis, and reported that none of those patients showed any signs of inflammatory reaction, rejection, or infection, nor abnormal results in blood tests. More recently, IP-CHA has been used as an autogenous bone graft substitute in oral and maxillofacial surgery cases (24). We previously reported implant treatment and maxillary sinus floor augmentation performed with a granular type of IP-CHA in a female patient, and those results showed a sufficient amount of osseointegration in the implant fixture, while histological analysis indicated that IP-CHA granules have strong potential to induce bone growth (24). In that case, we collected an autogenous bone block $(10 \times 8 \mathrm{~mm})$ from the maxillary tuberosity to prepare a graft comprised of a mixture of IP-CHA and cortical bone (Table I). We have also previously reported a clinical case of horizontal alveolar ridge atrophy following resection of a maxillary bone cyst, in which autogenous onlay bone grafting with IP-CHA granules was successfully used for prosthetic treatment (25). In that case, an autogenous block bone $(10 \times 15 \mathrm{~mm})$ was collected from the mandibular ramus, and a granular type of IP-CHA was applied to fill gaps between an autogenous bone block and host bone in order to restore bone volume (Table I). When a granular type of IP-CHA is applied as a substitute for bone augmentation, a residual bone wall or additional materials, such as titanium mesh, are needed to ensure space maintenance of the granules. For the present case, we used an IP-CHA block for onlay grafting in implant treatment without autogenous block bone grafting (Table I). At a follow-up examination performed 3 years 5 month after initial placement, the IP-CHA block could be observed on the alveolar bone, and the gap between it and the host bone showed a smooth transition, suggesting that 
IP-CHA improves integration with newly-formed bone tissue. At more than 5 years after the initial surgery, the implant and superstructure continued to show no problems. Application of an IP-CHA block can overcome disadvantages associated with autogenous bone block grafting, thus we consider it to be useful as a substitute for block bone grafting in patients undergoing implant treatment.

\section{Acknowledgements}

We express our deep appreciation for the late Professor Nobuyuki Kamata (Hiroshima University, Japan) for the excellent guidance regarding this case.

\section{References}

1. Chiapasco M,Zaniboni M and Boisco M: Augmentation procedures for the rehabilitation of deficient edentulous ridges with oral implants. Clin Oral Implant Res 17 (Suppl 2): S136-S159, 2006.

2. Pikos MA: Block autografts for localized ridge augmentation: Part I. The posterior maxilla. Implant Dent 8: 279-285, 1999.

3. Esposito M, Grusovin MG, Felice P, Karatzopoulos G, Worthington HV and Coulthard P: The efficacy of horizontal and vertical bone augmentation procedures for dental implants-a Cochrane systematic review. Eur J Oral Implantol 2: 167-184, 2009.

4. Clementini M, Morlupi A, Agrestini C and Ottria L: Success rate of dental implants inserted in autologous bone graft regenerated areas: A systematic review. Oral Implantol (Rome) 4: 3-10, 2012.

5. Donos N, Mardas N and Chadha V: Clinical outcomes of implantsfollowing lateral bone augmentation: Systematic assessment of available options (barrier membranes, bone grafts, split osteotomy). J Clin Periodontol 35 (8 Suppl): S173-S202, 2008.

6. Kuchler U and von Arx T: Horizontal ridge augmentation in conjunction with or prior to implant placement in the anterior maxilla: A systematic review. Int J Oral Maxillofac Implants 29 (Suppl): S14-S24, 2014.

7. Misch CM: Comparison of intraoral donor sites for onlay grafting prior to implant placement. Int J Oral Maxillofac Implants 12: 767-776, 1997

8. Stubinger S, Nuss K, Landes C, von Rechenberg B and Sader R: Harvesting of intraoral autogenous block grafts from the chin and ramus region: Preliminary results with a variable square pulse Er: YAG laser. Lasers Surg Med 40: 312-318, 2008.

9. Banwart JC, Asher MA and Hassanein RS: Iliac crest bone graft harvest donor site morbidity. A statistical evaluation. Spine (Phila Pa 1976) 20: 1055-1060, 1995.

10. Yoshikawa H, Tamai N, Murase T and Myoui A: Interconnected porous hydroxyapatite ceramics for bone tissue engineering. J R Soc Interface 6 (Suppl 3): S341-S348, 2009.

11. Yoshikawa $\mathrm{H}$ and Myoui $\mathrm{A}$ : Bone tissue engineering with porous hydroxyapatite ceramics. J Artif Organs 8: 131-136, 2005.
12. Minami M, Takechi M, Ohta K, Ohta A, Ninomiya Y, Takamoto M, Fukui A, Tada M and Kamata N: Bone formation and osseointegration with titanium implant using granular- and block-type porous hydroxyapatite ceramics (IP-CHA). Dent Mater J 32: 753-760, 2013.

13. Misch CM: Comparison of intraoral donor sites for onlay grafting prior to implant placement. Int J Oral Maxillofac Implant 12: 767-776, 1997.

14. Pourabbas R and Nezafati S: Clinical results of localized alveolar ridge augmentation with bone grafts harvested from symphysis in comparison with ramus. J Dent Res Dent Clin Dent Prospect 1: $7-12,2007$.

15. Stellingsma C, Vissink A, Meijer HJ, Kuiper C, Raghoebar GM and Raghoebar GM: Implantology and the severely resorbed edentulous mandible. Crit Rev Oral Biol Med 15: 240-248, 2004.

16. Bucholz RW, Carlton A and Holmes R: Interporous hydroxyapatite as a bone graft substitute in tibial plateau fractures. Clin Orthop Relat Res: 53-62, 1989.

17. Holmes RE, Bucholz RW and Mooney V: Porous hydroxyapatite as a bone graft substitute in diaphyseal defects: A histometric study. J Orthop Res 5: 114-121, 1987.

18. Uchida A, Araki N, Shinto Y, Yoshikawa H, Kurisaki E and Ono K: The use of calcium hydroxyapatite ceramic in bone tumour surgery. J Bone Joint Surg Br 72: 298-302, 1990.

19. Ayers RA, Simske SJ, Nunes CR and Wolford LM: Long-term bone ingrowth and residual micro hardness of porous block hydroxyapatite implants in humans. J Oral Maxillofac Surg 56: 1297-1302, 1998

20. Tamai N, Myoui A, Tomita T, Nakase T, Tanaka J, Ochi T and Yoshikawa H: Novel hydroxyapatite ceramics with an interconnective porous structure exhibit superior osteoconduction in vivo. J Biomed Mater Res 59: 110-117, 2002.

21. Tamai N, Myoui A, Kudawara I, Ueda T and Yoshikawa H: Novel fully interconnected porous hydroxyapatite ceramic in surgical treatment of benign bone tumor. J Orthop Sci 15: 560-568, 2010.

22. Doi K, Oue H, Morita K, Kajihara S, Kubo T, Koretake K, Perrotti V, Lezzi G, Piattelli A and Akagawa Y: Development of implant/interconnected porous hydroxyapatite complex as new concept graft material. PLoS One 7: e49051, 2012.

23. Shi K, Hayashida K, Hashimoto J, Sugamoto K, Kawai H and Yoshikawa $\mathrm{H}$ : Hydroxyapatite augmentation for bone atrophy in total ankle replacement in rheumatoid arthritis. J Foot Ankle Surg 45: 316-321, 2006

24. Shigeishi H, Takechi M, Nishimura M, Takamoto M, Minami M, Ohta K and Kamata N: Clinical evaluation of novel interconnected porous hydroxyapatite ceramics (IP-CHA) in a maxillary sinus floor augmentation procedure. Dent Mater J 21: 54-60, 2012.

25. Kubozono K, Takechi M, Ohta K, Ono S, Nakagawa T, Fujimoto $S$ and Kamata N: Aesthetic recovery of alveolar atrophy following autogenous onlay bone grafting using interconnected porous hydroxyapatite ceramics (IP-CHA) and resorbable poly-L-lactic/polyglycolic acid screws: Case report. BMC Oral Health 14: 60, 2014. 